\title{
Plants with evidence-based therapeutic effects against neurodegenerative diseases
}

\begin{abstract}
Neurodegenerative diseases are a group of illnesses, which affect both central and peripheral nervous system inducing physiological dysfunctions related with loss of movements and mental integrity, among others. In general, these diseases are incurable and only few drugs may alleviate the symptomatology or prolong the survival of patients. In this setting, medicinal plants might constitute adjuvant therapies with conventional pharmacotherapeutics in order to reduce or remedy the neurodegenerative illness. The aim of this review is to present the neuropharmacological activity of some phytochemicals from noteworthy medicinal plants that have been used in traditional medicine. Particularly, we will focus on medicinal plants with evidence-based therapeutic effects against neurodegenerative diseases.

Crucial among these plants is Daphne Genkwa, with its active components genkwanine N and yuanhuacin, which are both able to activate the transcriptional function of the Nurr1 receptor. Another plant to be highlighted is Jatropha multifida containing apocynin, a selective inhibitor of the phagocyte NADPH oxidase. Galanthus nivalis is a remarkable plant for its content in galantamine, an inhibitor of the acetylcholinesterase enzyme and an inhibitor of the nicotinic acetylcholine receptor (nAChR) with a mechanism consistent with open-channel pore blockade. Galantamine is currently used in Alzheimer's disease and dementias. Ginkgo biloba is known for its leaves as a natural source of flavonoids and polyphenolic compounds and it is used in the treatment of progressive neurodegenerative disorders such as Alzheimer's disease. Glycine max or soybeans contain isoflavones such as genistein, daidzein and glycitein. Among these phytochemicals, genistein should be underlined for its potential to alleviate neurological symptoms present in most lysosomal storage diseases by decreasing the biosynthesis of accumulating substrates.
\end{abstract}

Keywords: genkwanine N, galantamine, genistein, Ginkgo biloba, neurodegenerative diseases
Volume 7 Issue 5 - 2019

\section{González MP, Benedí J, Bermejo-Bescós P, Martin-Aragon S \\ Departamento de Farmacología, Farmacognosia y Botánica,}

Facultad de Farmacia, Universidad Complutense, Spain

Correspondence: Sagrario Martin-Aragon, PhD. Departamento de Farmacología, Farmacognosia y Botánica, Facultad de Farmacia, Universidad Complutense, Plaza Ramón y Cajal s/n, 28040 Madrid, Spain,Tel+349l 39417 67, Email smartina@ucm.es

Received: August 02, 2019 | Published: September 24, 2019
Abbreviations: $\mathrm{A} \beta, \beta$-amyloid; $\mathrm{AChE}$, acetylcholinesterase enzyme; $\mathrm{AD}$, Alzheimer's disease; ALS, amyotrophic lateral sclerosis; AMPA, $\alpha$-Amino-3-hydroxy-5-methyl-4-isoxazolepropionic acid; ApoE, human Apolipoprotein-E; APP, amyloid precursor protein; $\mathrm{BH} 4$, tetrahydrobiopterin; $\mathrm{CaMKII}, \mathrm{Ca} 2+-$ and calmodulin-dependent protein kinase II; CREB, cyclic adenosine monophosphate response element-binding protein; GAG, glycosaminoglycans; EGb 761, a standardized Ginkgo biloba extract; GCH1, GTP cyclohydrolase I; GSK-3, glycogen synthase kinase-3; HD, Huntington's disease; hIAPP, human islet amyloid polypeptide; $\mathrm{H} 2 \mathrm{O} 2$, hydrogen peroxide; INF- $\gamma$, interferon- $\gamma$; IL-1, interleukin-1; IL-6, interleukin-6; IL- $\beta$, interleukin- $\beta$; JNK, c-Jun N-terminal kinase; LPS, lipopolysaccharide; MPO, myeloperoxidase; MPTP, 1-methyl-4-phenyl-1,2,3,6tetrahydropyridine; $\mathrm{nAChR}$, nicotinic acetylcholine receptor; $\mathrm{NF \kappa B}$, nuclear factor kappa B; NMDA, N-methyl-D-aspartate; NOX2, NADPH oxidase enzyme; NR4A2, nuclear receptor subfamily 4 group A member 2; Nurr1, nuclear receptor related 1 protein; polyQ, proteins containing glutamine repeats; ROS, reactive oxygen species; SOD1, superoxide dismutase 1; TBP, TATA box-binding protein; TFEB, transcription factor EB; TH, tyrosine hydroxylase; TLR4, tolllike receptor 4 ; TNF- $\alpha$, tumor necrosis factor $\alpha$

\section{Introduction}

Phytochemicals are chemical compounds produced by plants, generally to help them thrive or defend from competitors, predators or pathogens. Numerous phytochemicals may have curative properties because herbs and plants have been used to cure all sorts of illnesses for centuries. To establish an efficient therapy to protect or treat a disease it is necessary to know both the dysfunction of the disease and the characteristics of the active agent. To date, most drugs used are synthesized compounds whose structures are based on active components from plants. Although the knowledge of neurology dates back to prehistoric times, the real advances in this discipline occurred in the Renaissance and its knowledge has been widely advanced until now.

Neurodegeneration is the progressive loss of structures and functions and eventually the death of neurons. To date, neurodegenerative diseases are incurable and share numerous similarities. There is a great parallelism between different neurodegenerative dysfunctions including atypical protein assemblies (Alzheimer, Corea, Parkinson, prion diseases) as well as cellular death (Parkinson, Corea) $)^{1,2}$ and nervous demyelination (Multiple sclerosis, Amyotrophic lateral sclerosis). The discovery of these similarities offers a great help for therapeutic advances that might prevent or reduce the progress or disturbances of these diseases. Taken all this into account we must consider that the neurodegenerative diseases share similar dysfunctions as cellular death, inflammation and oxidative stress, resulting from specific mechanisms that might be target by certain phytochemicals. Moreover, the increasing life expectancy and the rising in the incidence of these disorders should urge the need for therapies to delay its progression. As for the rates of prevalence, 
it is intriguing that in the case of dementia, there is a growing consistency of reports indicating a potential decrease ${ }^{3,4}$ not ascribed to better treatment. This effect is probably the result of changes in lifestyle, which includes chronic consumption of plants and/or plantderived products containing phytochemicals with antioxidative, anti-amyloidogenic, anti-inflammatory and anti-apoptotic properties, among others.

Plants contain a great number of active components that may have an application in medicine although many of them have been synthetized by the pharmaceutical industry. In the developed countries, plant-derived drugs may be important, and, plant extracts and active principles prepared from plants are quite a lot representing a potential therapeutic interest. ${ }^{5}$ In the preset review, we aimed to outline the main molecular mechanisms responsible of the dysfunctions that occurred in the phenomena of neurodegeneration as well as the evidence-based mechanisms of action of particular phytochemical compounds in order to suggest and propose rational and consistent preventive therapies. We have not described separately the different active principles from a certain plant but the plants that may have some application in the therapy of neurodegenerative diseases due to its active constituents.

\section{Daphne genkwa}

Daphne genkwa is a plant species from the Thymelaeceae family and is one of the main herb used by the traditional Chinese medicine known as "yuan huä". The flowers buds of this plant are use with diuretic, antitussive, expectorant and antitumor purposes. But, beside that a methanol extract of the roots of this plant activated the transcriptional function of Nurr1, an orphan nuclear receptor that is essential for the differentiation ${ }^{6-10}$ and maintenance of dopaminergic neurons in the brain ${ }^{6}$ what may signify a therapeutic target for Parkinson' and other neurodegenerative diseases. The active components isolated from Daphne genkwa have been identified as genkwainine $\mathrm{N}$ and yuanhuavin. Both compounds have shown to enhance the function of Nurr 1 and inhibit the neuronal death induced by 6-hydoxydopamine on SH-SY5Y cells and, in addition, they have proved a beneficial effect on the neuroinflammation mediated by lipopolysaccharide (LPS) in BV-2 microglial cell line. ${ }^{7}$ Nurr1 inhibits the expression of proinflammatory genes in microglia and astrocytes. The repression of inflammation by Nurr1 activation decreases the loss of dopaminergic neurons in vivo and in vitro models, ${ }^{10}$ signifying a protective mechanism in Parkinson's disease.

It is known that the human NR4A2 gene encodes Nurr1 and, although its ligand is not well identified, it is known that Nurr1 protein is a member of the nuclear receptor family of the intracellular transcription factor, which plays a role in maintaining the dopaminergic system in the brain. This lies on the fact that Nurr1 abnormalities turn into damage of the function of dopaminergic system causing Parkinson's disease and a wide range of inflammatory and neurological diseases, including rheumatoid arthritis, schizophrenia and manic depression. Nurr1 is expressed in developing and mature dopaminergic neurons and is required for both its survival and its complete differentiation. ${ }^{7,11}$ Nurr1 also enhances the transcription of tyrosine hydroxylase (TH), the limiting enzyme of dopamine biosynthesis as well as of the GTP cyclohydrolase I (GCH1), the first enzyme in the biosynthesis of tetrahydrobiopterin (BH4), which is an essential factor to TH activity. ${ }^{12,13}$

Genkwanine and yuanhuacin obtained from extracts of Daphne genkwa activate the nuclear receptor Nurr1. This receptor is bound to different regions (sequences) of DNA from the cell nucleus and may actuate as transcriptional activator or transcriptional repressor depending on the cellular context. As a transcriptional activator may induce different survival signals which maintain the survival and development of dopaminergic neurons. Nurr1 also may enhance the transcription of both tyrosine hydroxylase and GTP-cyclohydrolase I enzyme. Reduced Nurr1 expression might result in dysfunction and progression of degenerative changes of dopaminergic neurons. The availability of molecules capable of activating this receptor might provide a promising therapy for neurodegenerative diseases, mainly Parkinson's disease. Due to the action of the active ingredients (genkwanine $\mathrm{N}$ and yuanhuacin of Daphne genkwa) on Nurr1 functions, one may suggest that this plant may exert neuroprotective, neurogenic or anti-inflammatory effects.

\section{Jatropha multifida}

Jatropha multifida is a plat species from the family Euphorbiaceae and is a shrub or treelet. The genus Jatropha comprises about 170 species of woody trees, shrubs, subshrubs or herbs. They are used in medicine to cure several diseases in Africa, Asia and Latin America. Plants extracts are reported for cytotoxicity, tumor-promoting, antimicrobial, antiprotozoal, anticoagulant, immunomodulating, antiinflammatory, and antioxidant properties and acetylcholinesterase inhibitors. $^{14-16}$

All parts of this plant, but mainly the seeds, have strong purgative properties and are insect repellents. Jatropha multifida contains apocynin (4'-hydroxy-3'-methoxyacetophenone or acetovanillone), a selective inhibitor of the phagocyte NADPH oxidase (NOX2 enzyme) which produces reactive oxygen species (ROS) being a well know element in host defence. Besides, there is evidence for a regulatory role of NOX2 in adaptative immunity suggesting a role in autoimmune diseases. ${ }^{17}$ Regarding this point, there is sufficient evidence suggesting that inflammatory mediators have a significant influence on the pathogenesis of neurodegenerative diseases what may propose apocynin as a potential treatment of neurodegenerative diseases because microglia cells are the main resource of NOX2 in the brain. This enzyme, when activated, induces reduction of $\mathrm{O}_{2}$ to superoxide $\left(\mathrm{O}_{2}^{-}\right)$. Superoxide may kill bacteria and fungi by mechanisms that are not yet fully understood. ${ }^{18}$ It may also forms hydrogen peroxide spontaneously that undergoes further reactions to generate $\mathrm{H}_{2} \mathrm{O}_{2}$ other ROS formation that can be used to kill bacteria and fungi by the immune system and to inevitably drive cell death mediating neurodegenerative brain injury.

When this enzyme is inhibited by apocynin, ROS formation is prevented and then neurodegeneration does not occur. The mechanism by which apocynin induces its action is not well known. However, apocynin is a selective inhibitor of NADPH oxidase and therefore it may prevent the production of superoxide in human white blood cells or neutrophils without interfering with the defences of the immune system. This might be due to the fat that apocynin is not an inhibitor of the NADPH oxidase from immune system cells as it occurs in the case of the NADPH oxidase from vascular smooth muscle cells. ${ }^{19}$ Apocynin mechanism from experimental studies may be summarized as follows:

1. Apocynin is metabolically activated by $\mathrm{H}_{2} \mathrm{O}_{2}$ in a myeloperoxidase (MPO-reaction) forming a symmetrical dimmer, dyapocynin ${ }^{20}$

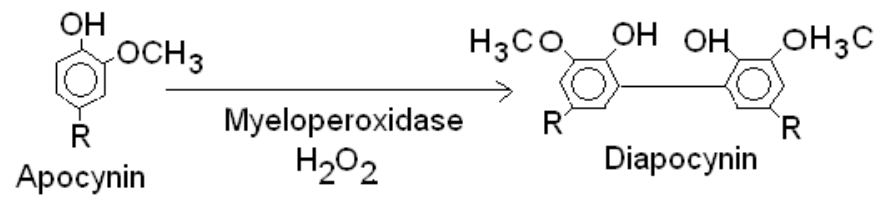

2. Diapocynin inhibits the superoxide production mediated by NOX2. However, diapocynin mays directly inhibit NOX2 regardless of MPO contribution. 
One important issue is that microglia cells not only express NOX2 but also secrete MPO after activation what might potentiate the apocynin action.

The pharmacological profile and low toxicity of apocynin might be considered for its use as a neuroprotective treatment. In this sense, some researchers have found that NOX2 inhibition promotes antiinflammatory microglial activation during neuroinflammation. ${ }^{21}$ This may imply that suppression of NOX2 with apocynin might restore microglia healthy. Other authors have shown that apocynin decreases the production of IL-1, TNF- $\alpha$ and nitric oxide by microglia. However, although the exact inductor of microglia activation in neurodegenerative disease is still uncertain it could be of interest should be note that glutamate toxicity requires NOX2 activation and that this may be inhibited by apocynin. ${ }^{22}$ On the other hand remarkable effects of apocynin have been observed in mouse models of neurodegenerative diseases such as amyotrophic lateral sclerosis (ALS) pathology whose motor neurons containing aggregates of superoxide dismutase 1 (SOD1) are hallmarks of ALS caused by mutations in the gene encoding SOD1. Harraz et al. ${ }^{23}$ have observed that oral administration of apocynin in drinking water to mutated SOD1 mice prolonged animal survival and delayed motor defects. They also observed that ALS mice treated with apocynin significantly increased their average lifespan. However, although this study was promising, other studies performed with the same mutants indicated that apocynin has not this beneficial effects. ${ }^{24}$

Other authors have administered apocynin as a daily dose via drinking water to a transgenic mouse model of Alzheimer's disease and observed a significant reduction of amyloid plaque size within cortex and hippocampus and a reduction of microglia content in cortex but not in hippocampus. However, this treatment could not imply any improvement in the tests of spatial memory. ${ }^{25}$ Apocynin may be extracted from different plants as Apocynum cannabinum and it has been identified as an active substance in the roots of Picrorhiza kurroa (Scrophulariaceae), a perennial plant from alpine Himalaya

\section{Galanthus nivalis}

Galanthus nivalis is a plant species from the Amaryllidaceae family that contains galantamine, an isoquinoline alkaloid produced by the plant. It was first discovered in 1958 by the Bulgarian Chemist D. Paskov and it was firstly used in the poliomyelitis treatment and the neuropathic pain and as an anesthetic. ${ }^{26}$ Nowadays, galantamine is obtained from bulbs of Galanthus woronowi Losinsk and Galanthus alpines and by synthesis. Galantamine has a unique dual mode of action affecting the cholinergic system as a reversible inhibitor of the acetylcholinesterase (AChE) enzyme. ${ }^{27}$ In concentrations ranging from $10 \mathrm{nM}$ to $1 \mu \mathrm{M}$, galantamine did not display direct modulatory effects at any receptor combination tested. At concentrations from $10 \mu \mathrm{M}$ and above, galantamine inhibits AChE activity through a mechanism consistent with open-channel pore blockade at all receptor types. ${ }^{28}$ Galantamine may cross the blood-brain barrier making it a condition to be used for neurological diseases. Galantamine also prevents mitochondrial dysfunctions protecting mitochondrial membrane potential due to its antioxidative properties..$^{29}$ Galantamine is currently used in Alzheimer's and dementia disease ${ }^{30}$ and its effectiveness has been confirmed in several clinical trials. In these patients, galantamine might achieve the efficiency of cholinergic system helping improving the cognitive function. Galantamine may also potentiate antioxidative activity $^{31}$ and prevent memory extinction in guinea pig exposure to organophosphorus pesticides. Galantamine may improve the AMPA receptor-mediated signalling, which could be neuroprotective improving memory in schizophrenia. Combination of galantamine and memantine (an antagonist of NMDA glutamate receptor) improves cognition in schizophrenia. ${ }^{32}$ In addition, galantamine could improve cognitive and behavioural symptoms in Alzheimer's disease and may have disease-modifying and neuroprotective properties. Moreover, galantamine could induce amyloid clearance ${ }^{33}$ mediated via stimulation of microglial nicotinic acetylcholine receptors. ${ }^{34}$

In the 1960 and 1970 the drug produced from Galanthus nivalis was commercialised under the name "Nivalin" being used in the Eastern European to treat polio and increase neurotransmission in the brain. However, this drug was more widely recognised in 1980 and used in the Alzheimer's disease treatment in 1990s.

\section{Ginkgo biloba}

Ginkgo biloba is a unique species of tree in the world that belongs to the Ginkgoaceae family. It is native to China and it is named as "yin xing". It is to Engelbert Kaempfer (1651-1716), a German scientist that the first description of the ginkgo and the name of the arboreal genus is due. Ginkgo biloba has been used in the traditional Chinese medicine and recently, it has begun to be used in the United States to treat physical and cognitive disorders related with age. ${ }^{35}$ Ginkgo biloba possesses a great variety of biological and pharmacological activities. The leaf extract of Ginkgo biloba (EGb761) has been used for years to treat memory deficits, including Alzheimer's disease and dementia ${ }^{36}$ and Parkinson's disease. ${ }^{37,38}$ Extracts of Ginkgo biloba leaves are a natural source of flavonoids and polyphenolic compounds that can stimulate the expression of genes related with antioxidant enzymes. The most benefits associated with the use of food rich in polyphenols include a reduced risk of coronary diseases and prevention of cancer and some neurodegenerative diseases. ${ }^{39-41}$

Ginkgo biloba extract is obtained from green leaf and has several properties as phychoanaleptic against dementia. It contains two bioactive components, a glucoside flavonoide and a lactone from terpenes (trilactone), denominated ginkgolides and bilobalide. ${ }^{42}$ It contains ginkgolic acid $\mathrm{A}, \mathrm{B}$, and $\mathrm{C}$ with allergenic properties. It is believed that the therapeutic mechanism is due to its antioxidant and antiedema action. The EGb 761 extracts from leaf possess neuroprotective properties and have been used in the treatment of progressive neurodegenerative disorders in Alzheimer's disease. ${ }^{43}$ It has also been shown that Ginkgo bioactive agents as ginkgolides and bilobalide exert beneficial effects in animal models of acute neurodegeneration induced mainly by hypoxia and ischemia. ${ }^{44-46}$ Mdzinarishvili et al., ${ }^{47}$ showed that pre-treatment with EGb761 extracts strongly reduce cellular oedema formation and neurodegeneration under ischemia conditions and that the mechanism could be related to a reduction of excitotoxicity due to high glutamate release during ischemia.

Huang et al., ${ }^{48}$ observed suppression of excitotoxicity and apoptosis by treatment with extract EGb761 from Ginkgo biloba in a model of type 17 spinocerebellar ataxia. This is a polyglutamine disease caused by the expansion of $\mathrm{CAG} / \mathrm{CAA}$ repeated groups in the TATA box-binding protein (TBP) gene that leads to protein aggregates in these patients. This founding suggests that EGb761 may be a potential therapeutic agent for treatment of this disease. Using a cellular model of Huntington's disease (HD) by employing cells expressing pathological variants of a polyglutamine protein (polyQ protein), some authors have shown that the EGb761 extract modulates proteasome formation by inducing proteasomal protein degradation suggesting a therapeutic function of this extract in neurodegenerative disorders with disturbances in protein homeostasis.

It has been also shown that in Alzheimer's disease the treatment with EGb761 induces protective effect under distinct mechanisms, such as: 1) antioxidative; ${ }^{49,50}$ 2) scavenging of free radicals, ${ }^{51} 3$ ) antiamyloidogenic, ${ }^{52,53}$ 4) anti-inflammatory; ${ }^{54}$ and 5) anti-apoptotic. ${ }^{55}$ Although the mechanism of action of EGb761 extracts from Ginkgo biloba is not well known several reports may be taken into account. It has been found that bilobalide improves mitochondrial activity and 
preserves ATP level during ischemia. ${ }^{56}$ Schwarzkopf et al. ${ }^{57}$ concluded that neuroprotection by bilobalide may involve a mechanism in which this compound reverses ischemia-induced changes in mitochondria, leading to a reduction of glutamate release. Other constituents of EGb761, as flavonoids and ginkgolides, seem to have beneficial effect in models of neurodegenerative diseases. ${ }^{58}$

Li et al. ${ }^{59}$ have studied the pharmacological action of YY-1224, a terpene trilactone extract of Ginkgo biloba, against $\beta$-amyloid (A $\beta)$ toxicity and found that the protective effects of YY-1224 against A $\beta$ toxicity may be associated with the inhibition of the $A \beta$-mediated pro-inflammation of microglia as well as with its neurotrophic and antioxidant effects. Some authors have proven that EGb761 reduces neuronal apoptosis and promotes angiogenesis in experimental intracerebral haemorrhage via RSK1/GSL3 $\beta$ pathway. ${ }^{57}$ It has been observed that the neuroprotection accounted for EGb761 derivedflavonoid monomers in an in vitro three-dimensional stem cellderived neural model was mainly related to its antioxidant effect and inhibition of caspase 3 activity. ${ }^{60}$

\section{Genista tinctoria}

Genista tinctoria is a flowering plant that belongs to Leguminosae (Fabaceae) family, gender Genista. It is native to meadows and pastures in Europe and Turkey. This plant was used from ancient times for producing a yellow dye. This plant contains an isoflavone, which was first isolated from this plant in 1899 and was denominated genistein. The medicinal parts are the flowering twing and it has been used in popular medicine in skin diseases and other uses, even in modern times. Although, the identification, in this plant, of a yellow dye has led to be widely used in textile industry, however, genistein is an isoflavone with a great therapeutic use. In nature, genistein is found in several edible plants as lupin, soy, beans, coffee and so on $^{61-63}$ and medical plants as Flemingia vestita ${ }^{64}$ and Flemingia macrophylia. Genistein is one of the major isoflavones in soybeans with phytoestrogenic activities and may contribute to its potential antiinflammatory, anticarcinogenic and hypocolesterolemic effects. ${ }^{65-69}$ Genistein is bound to the cell to an estrogenic receptor beta agonist and represents an alternative to oestrogen in treating neurodegenerative disorders. Due to its estrogenic nature, genistein has a mechanism through its bound to estrogenic receptors localized in the cellular nucleus promoting activation and deactivation of determinate genes regulators of the protein synthesis. ${ }^{70,71}$ The are numerous evidences supporting the protective effect of genistein in ischemia. ${ }^{72}$ Soy isoflavones have several uses not only as endogenous estrogens in hormonal pathologies but also in inflammatory neurodegenerative diseases and pain. ${ }^{73}$ Soy contains isoflavones like genistein, diadzein ${ }^{74}$ and glycitein, an $o$-methylated isoflavone that presents a weak estrogenic activity. ${ }^{75}$ Isoflavones are polyphenol compounds with phytoestrogen activity and involved as potential beneficial factor in cardiovascular diseases and other actions as protectors in the repair of the brain injury in rats. ${ }^{76,77}$ Several studies on the cognitive effects of soy isoflavone have been reported presenting benefit, ${ }^{78}$ no effect ${ }^{79}$ and possible harm ${ }^{80}$ in older patients with difficulties in memory.

It has been proposed that genistein (5,7-dihydroxy-3-(4hydroxyphenil9-4H-1-benzopyran-4-one) may be a potential drug for therapy of mucopolysaccharidoses, a group of metabolic diseases caused by mutations which lead to inefficient degradation of glycosaminoglycans (GAGs) and may cause neurological symptoms. This disease may be detected by the presence of heparin sulphate in urine and may be cause of different neurology symptoms. It has been demonstrated that genistein can cross the blood-brain barrier, making it a potential drug for the treatment of neurological symptoms present in most lysosomal storage diseases. Malinowska et al., ${ }^{81}$ observed that in neurodegenerative metabolic disorders, such as mucopolysaccaridosis III B or Sanfilippo disease, genistein administered at high doses to
MPSIIIB mice, significantly reduces lysosomal storage of GAGs, heparin sulphate substrate and neuroinflammation in the cerebral cortex and hippocampus, resulting in correction of behaviour defects. The mechanism by which genistein make its action is not well known. Genistein aglycone is a non-specific inhibitor of tyrosine kinase ${ }^{82,83}$ at pharmacological doses and reduces GAG substrate which is accumulated in fibroblasts of several mucopolysaccharidosis. ${ }^{84}$ Genistein administered to MPS IIIB mice significantly reduces liver lysosomal storage ${ }^{85}$ and inhibits lipopolysaccharidosis induced by $\mathrm{TNF} \alpha$ in astrocyte cultures ${ }^{86}$ and protects dopaminergic neurons by inhibiting microglial activation. ${ }^{87}$ Moskot et al., ${ }^{88}$ observed that the molecular mechanism of genistein action involves not only the GAG synthesis but the lysosomal metabolism by activating the transcription factor EB (TFEB). TFEB has been demonstrated as a crucial regulator of lysosomal biogenesis and autophagy. Accumulated studies suggest that TFEB has beneficial effects on neurodegenerative diseases, and also regulates cell clearance in lysosomal storage disorders. ${ }^{89}$ Ren et al.,90 observed that genistein has an anti-aggregant effect on both amyloid $\beta$-peptide associated with Alzheimer's disease and human islet amylin (hIAPP) associated with type 2 diabetes. Park et al., ${ }^{91}$ found a protective effect of genistein against neuronal degeneration in $\mathrm{ApoE}^{-/-}$mice demonstrating that genistein induced significant inhibition of oxidative stress and alleviated neuroinflammation. Genistein supplementation also reduced $A \beta$-deposition by reducing gene expression of APP-processing enzymes and decreased tau hyperphosphorylation by inactivating GSK-3 and JNK in the hippocampus and cortex and hyperphosphorylation in $\mathrm{ApoE}^{-/}$mice fed with a high diet in genistein. This study also provides that dysregulated cholesterol metabolism may lead to an accumulation of $\mathrm{A} \beta$ and increases in oxidative stress and neuroinflammation.

It is increasing the knowledge that obesity, diabetes mellitus, hypercholesterolemia and non-alcoholic steatohepatitis are associated with multiple aspects of brain pathogenesis. ${ }^{92}$ It is believed that altered cholesterol metabolism play a role in major pathophysiological neurodegenerative diseases and some studies have demonstrated that the absence in apolipoprotein E (ApoE), the main apolipoprotein released by astrocytes and microglia that transports cholesterol between glial cells and neurons,${ }^{93}$ leads to an increased susceptibility to neurodegeneration.

Yu et al. ${ }^{94}$ found neuroprotection mediated by genistein in cortical neurons treated with $A \beta$-amyloid 25-35. These authors observed that genistein protected cortical neurons against apoptosis and maintained mitochondrial function damaged by A $\beta$-amyloid 25-35. Xi et al., ${ }^{95}$ observed that SH-SY5Y cells incubated with A $\beta$-amyloid 25-35 induced down regulation of synaptophysin and postsynaptic marker as NR1 and NR2B which are subunits of the NMDA glutamate receptor. The author also detected that $A \beta$-amyloid 25-35 induced, on these cells, down regulation of mRNA and protein expression of $\mathrm{Ca}^{2+} /$ calmodulin, CaMKII/pCaMKII and CREB/pCREB. However, pre-treatment with genistein reversed all these effects indicating that genistein mediated this action with the regulation of synaptic markers and activation of $\mathrm{CaM} / \mathrm{CaMK} / \mathrm{CREB}$ signalling pathway.

McDowell et al., ${ }^{96}$ found that genistein prevented apoptosis in cultured ventral spinal cords cells exposure to supernatant of activated microglia by INF- $\gamma$. This protection was associated with increased expression of estrogen receptor $\beta$ suggesting that genistein may promote neuroprotection via receptor-mediated pathways. Ma et al. ${ }^{97}$ found that genistein may induce neuroprotective effect against action of A $\beta$-amyloid 25-35 in C6 cells. When these cells were treated with $A \beta$-amyloid 25-35 there was a loss of cell viability, increase of TNF $\alpha$ and IL- $\beta$, inflammatory markers, as well as upregulation of mRNA and protein expression of TLR 4 and I $\kappa B$. When the treatment was performed in presence of genistein there was an increase in cell viability, decrease of inflammatory markers (TLR4 and IL- $\beta$ ) and 
reversion of the deregulation of TLT4 and Iк B. From these results, these authors inferred that the action of genistein might be mediated through the regulation of TLR4/NFKB signalling pathway and the abolition of inflammatory response.

Beneficial actions of genistein on mitochondrial DNA damage induced by A $\beta$-amyloid 25-35 in C6 glioma cells has been detected by $\mathrm{Ma}$ et al., ${ }^{98}$ confirming that genistein could alleviate the mitochondrial-targeted oxidative damage induced by A $\beta$-amyloid 2535. This finding suggests to the authors that genistein may be used by the treatment of neurodegenerative diseases. Liu et al., ${ }^{99}$ using a model of rat ovariectomized to which was induced Parkinson by treatment with MPTP, observed significant decrease in dopamine and dihydroxyphenylacetic acid, a dopamine metabolite, and homovanillic acid in the striatum. This action was restore with genistein or estrogen pretreatment indicating that genistein may have neuroprotective effect on dopaminergic neurons.

In neurodegenerative diseases, genistein may have different effects such as estrogenic, anti-inflammatory, antioxidant and antiaggregating. The estrogenic effect is mediate through the binding of genistein on nuclear estrogenic receptor promoting gen regulators of protein synthesis. The anti-inflammatory action is mediate by regulation of inflammatory markers as TLR4 and IL-6. The antioxidant action is promoted by the mitochondrial protection mediated by genistein that decreases ROS formation. The anti-aggregating action is due to the action of genistein preventing the protein binding (aggregation) and, as consequence, avoiding the aggregated protein that may be the cause of cellular death.

\section{Conclusion}

We are concerned that the approach of the ethnobotanical research should change, as most research on medicinal plants emphasizes the scientific documentation of plants and its uses for the benefit of large transnationals. We claim with this review for an interdisciplinary approach of ethnobotany based on the spread of knowledge on the phytochemical principles and its mechanisms for preventing neurodegenerative disorders as a right of any member of society. What is more, within an ageing society, the knowledge of natural sources and its potential consumption for disease prevention should be disseminated as part of the health education.

\section{Acknowledgements}

Authors pay tribute to the human groups that have collaborated with both their traditional knowledge and collection of the plants reviewed.

\section{Conflicts of interest}

All authors declare no actual or potential conflicts of interest.

\section{References}

1. Rubinsztein DC. The roles of intracellular protein-degradation pathways in neurodegeneration. Nature. 2006;443(7113):780-786.

2. Bredesen DE, Rao RV, Mehlen P. Cell death in the nervous system. Nature. 2006;443(7113):796-802.

3. Skoog I, Börjesson-Hanson A, Kern S, et al. Decreasing prevalence of dementia in 85-year olds examined 22 years apart: the influence of education and stroke. Sci Rep. 2017;7(1):6136.

4. Aguirre-Milachay E, Alva-Díaz C. Is it possible to reduce the prevalence of dementia? New approaches are needed to deal with dementia. Semergen. 2018;44(8):586-589.
5. Mamczarz J, Kulkarni GS, Pereira EFR, et al. Galantamine counteracts development of learning impairment in guinea pigs exposed to the organophosphorus poison soman: Clinical significance. Neurotoxicology. 2011;32(6):785-798.

6. Han BS, Kim KS, Kim YJ, et al. Daphnane Diterpenes from Daphne genkwa Activate Nurr1 and Have a Neuroprotective Effect in an Animal Model of Parkinson's Disease. J Nat Prod. 2016;79(6):1604-1609.

7. Saucedo-Cardenas O, Quintana-Hau JD, Le WD, et al. Nurr1 is essential for the induction of the dopaminergic phenotype and the survival of ventral mesencephalic late dopaminergic precursor neurons. Proc Natl Acad Sci USA. 1998;95(7):4013-4018.

8. Chung S, Leung A, Han BS, et al. Wnt1-1mx 1 a forms a novel autoregulatory loop and controls midbrain dopaminergic differentiation synergistically with the SHH-FoxA2 pathway. Cell Stem Cell. 2009;5(6):646-658.

9. Kadkhodaei B, Ito T, Joodmardi E, et al. Nurr1 is required for maintenance of maturing and adult midbrain dopamine neurons. $J$ Neurosci. 2009;29(50):15923-15932.

10. Seiko K, Winner B, Carson CT, et al. Nurr1/CoREST pathway in microglia and astrocytes protects dopaminergic neurons from inflammationinduced death. Cell. 2009;137(1):47-59.

11. Zetterström RH, Solomin L, Jansson L, et al. Dopamine neuron agenesis in Nurr1-deficient mice. Science. 1997;276(5310):248-250.

12. Kim KS, et al. Orphan nuclear receptor Nurr1 directly transactivates the promoter activity of the tyrosine hydroxylase gene in a cell-specific manner. J Neurochem. 2003;85(3):622-634.

13. Gil M, McKinney C, Lee MK, et al. Regulation of GTP cyclohydrolase I expression by orphan receptor Nurr1 in cell culture and in vivo. $J$ Neurochem. 2007;101(1):142-150.

14. Anani K, Adjrah Y, Améyapoh Y, et al. Antimicrobial, Anti-inflammatory and Antioxidant Activities of Jatropha multifida L. (Euphorbiaceae). Pharmacognosy Res. 2016;8(2):142-146.

15. Hart BA, Copray S, Philippens I. Apocynin, a low molecular oral treatment for neurodegenerative disease. Biomed Res Int. 2014;2014:298020.

16. Rampadarath S, Puchooa D, Ranghoo-Sanmukhiya VM. Antimicrobial, phytochemical and larvicidal properties of Jatropha multifida Linn. Asian Pac J Trop Med. 2014;7S1:S380-S383.

17. Cachat J, Deffert C, Hugues S, et al. Phagocyte NADPH oxidase and specific immunity. Clin Sci. 2015;128(10):635-648.

18. Slauch JM. How does the oxidative burst of macrophages kill bacteria? Still an open question. Mol Microbiol. 2011;80(3):580-583.

19. Heumüller S, Wind S, Barbosa-Sicard E, et al. Apocynin is not an inhibitor of vascular NADPH oxidases but an antioxidant. Hypertension. 2008;51(2):211-217.

20. Johnson DK, Schillinger KJ, Kwait DM, et al. Inhibition of NADPH oxidase activation in endothelial cells by orthomethoxy-substituted catechols. Endothelium. 2002;9(3):191-203.

21. Choi SH, Aid S, Kim HW, et al. Inhibition of NADPH oxidase promotes alternative and anti-inflammatory microglial activation during neuroinflammation. J Neurochem. 2012;120(2):292-301.

22. Barger SW, Goodwin ME, Porter MM, et al. Glutamate release from activated microglia requires the oxidative burst and lipid peroxidation. $J$ Neurochem. 2007;101(5):1205-1213.

23. Harraz MM, Marden JJ, Zhou W, et al. SOD1 mutations disrupt redoxsensitive Rac regulation of NADPH oxidase in a familial ALS model. $J$ Clin Investig. 2008;118(2):659-670. 
24. Trumbull KA, McAllister D, Gandelman MM, et al. Diapocynin and apocynin administration fails to significantly extend survival in G93A SOD1 ALS mice. Neurobiol Dis. 2012;45(1):137-144.

25. Levesque LS, Surace MJ, Block ML, et al. Chronic apocynin treatment attenuates beta amyloid plaque size and microglial number in $\mathrm{hAPP}(751)$ SL mice. PLOS One. 2011;6(5):1-11.

26. Ng YP, Or TCT, Ip NY. Plant alkaloids as drug leads for Alzheimer's disease. Neurochem Int. 2015;89:260-270.

27. Albuquerque EX, Santos MD, Alkondon M, et al. Modulation of nicotinic receptor activity in the central nervous system: a novel approach to the treatment of Alzheimer disease. Alzheimer Dis Assoc Disord. 2001;15(Suppl 1):S19-S25.

28. Kowal NM, Ahring PK, Liao VWY, et al. Galantamine is not a positive allosteric modulator of human $\alpha 4 \beta 2$ or $\alpha 7$ nicotinic acetylcholine receptors. Br J Pharmacol. 2018;175(14):2911-2925.

29. Ezoulin MJ, Ombetta JE, Dutertre-Catella H, et al. Antioxidative properties of galantamine on neuronal damage induced by hydrogen peroxide in SK-N-SH cells. Neurotoxicology. 2008;29(2):270-277.

30. Lilienfeld S. Galantamine-a novel cholinergic drug with a unique dual mode of action for the treatment of patients with Alzheimer's disease. CNS Drug Rev. 2002;8(2):159-176.

31. Romero A, Egea J, Garcia AG, et al. Synergistic neuroprotective effect of combined low concentrations of galantamine and melatonin against oxidative stress in SH-SY5Y neuroblastoma cells. J Pineal Res. 2010;49(2):141-148

32. Koola MM, Buchanan RW, Pillai A, et al. Potential Role of the Combination of Galantamine and Memantine to Improve Cognition in Schizophrenia. Schizophr Res. 2014;157(1-3):84-89.

33. Bhattacharya S, Haertel C, Maelicke A, et al. Galantamine Slows Down Plaque Formation and Behavioral Decline in the 5XFAD Mouse Model of Alzheimer's Disease. PLOS One. 2014;9(2):1-12.

34. Takata K, Kitamura Y, Saeki M, et al. Galantamine-induced Amyloid- $\beta$ Clearance Mediated via Stimulation of Microglial Nicotinic Acetylcholine Receptors. J Biol Chem. 2010;285(51):40180-40191.

35. Doraiswamy PM. Non-cholinergic strategies for treating and preventing Alzheimer's disease. CNS Drugs. 2002;16(12):811-824.

36. Mohanta TK, Tamboli Y, Zubaidha PK. Phytochemical and medicinal importance of Ginkgo biloba L. Nat Prod Res. 2014;28(10):746-752.

37. El-Ghazaly MA, Sadik NA, Rashed ER, et al. Neuroprotective effect of EGb761 and low-dose whole-body $\gamma$-irradiation in a rat model of Parkinson's disease. Toxicol Ind Health. 2015;31(12):1128-1143.

38. Wang YQ, Wang MY, Fu XR, et al. Neuroprotective effects of ginkgetin against neuroinjury in Parkinson's disease model induced by MPTP via chelating iron. Free Radic Res. 2015;49(9):1069-1080.

39. Chen X, Zeng L. Ginkgo biloba extract 761 enhances 5-fluorouracil chemosensitivity in colorectal cancer cells through regulation of high mobility group-box 3 expression. Am J Transl Res. 2018;10(6):17731783.

40. Xu X, Guo S, Hao X, et al. Improving antioxidant and antiproliferative activities of colla corii asini hydrolysates using ginkgo biloba extracts. Food Sci Nutr. 2018;6(4):765-772.

41. Gargouri B, Carstensen J, Bhatia HS, et al. Anti-neuroinflammatory effects of Ginkgo biloba extract EGb761 in LPS-activated primary microglial cells. Phytomedicine. 2018;44:45-55.

42. Defeudis FV. Bilobalide and neuroprotection. Pharmacol Res. 2002;46(6):565-568.
43. Ramassamy C, Longpre F, Christen Y. Ginkgo bilobaa extract (EGb761) in Alzheimer's disease in there any evidence? Curr Alzheimer Res. 2007;4(3):253-262.

44. Pierre S, Jamme I, Droy-Lefaix MT, et al. Ginkgo biloba extract (EGb 761) protects $\mathrm{Na}, \mathrm{K}$-ATPase activity during cerebral ischemia in mice. Neuroreport. 1999;10(1):47-51.

45. Yang $\mathrm{X}$, Zheng $\mathrm{T}$, Hong $\mathrm{H}$ et al. Neuroprotective effects of Ginkgo biloba extract and Ginkgolide B against oxygen-glucose deprivation/ reoxygenation and glucose injury in a new in vitro multicellular network model. Front Med. 2018;12(3):307-318.

46. Yin B, Xu Y, Wei R, et al. Ginkgo biloba on focal cerebral ischemia: a systematic review and meta-analysis. Am J Chin Med. 2014;42(4):769783.

47. Mdzinarishvili A, Sumbria R, Lang D, et al. Ginkgo Extract EGb761 Confers Neuroprotection by Reduction of Glutamate Release in Ischemic Brain. J Pharm Pharm Sci. 2012;15(1):94-102.

48. Huang DS, Lin HY, Lee-Chen GJ, et al. Treatment with a Ginkgo biloba extract, EGb 761, inhibits excitotoxicity in an animal model of spinocerebellar ataxia type 17. Drug Des Devel Ther. 2016;10:723-731.

49. Horáková L, Licht A, Sandig G, et al. Standardized extracts of flavonoids increase the viability of PC12 cells treated with hydrogen peroxide: effects on oxidative injury. Arch Toxicol. 2003;77(1):22-29.

50. Kumar A, Singh A. A review on mitochondrial restorative mechanism of antioxidants in Alzheimer's disease and other neurological conditions. Front Pharmacol. 2015;6:206.

51. Maitra I, Marcocci L, Droy-Lefaix MT, et al. Peroxyl radical scavenging activity of Ginkgo biloba extract EGb 761. Biochem Pharmacol. 1995;49(11):1649-1655.

52. Brunetti L, Orlando G, Menghini L, et al. Ginkgo biloba leaf extract reverses amyloid $\beta$-peptide-induced isoprostane production in rat brain in vitro. Planta Medica. 2006;72(14):1296-1299.

53. Wang YQ, Wang MY, Fu XR, et al. Neuroprotective effects of ginkgetin against neuroinjury in Parkinson's disease model induced by MPTP via chelating iron. Free Radic Res. 2015;49(9):1069-1080.

54. Liu X, Hao W, Qin Y, et al. Long-term treatment with Ginkgo biloba extract EGb 761 improves symptoms and pathology in a transgenic mouse model of Alzheimer's disease. Brain Behav Immun. 2015;46:121-131.

55. Smith JV, Burdick AJ, Golik P, et al. Anti-apoptotic properties of Ginkgo biloba extract EGb 761 in differentiated PC12 cells Cell Mol Biol. 2002;48(6):699-707.

56. Pierre S, Jamme I, Droy-Lefaix MT, et al. Ginkgo biloba extract (EGb 761) protects $\mathrm{Na}, \mathrm{K}-\mathrm{ATPa}$ e activity during cerebral ischemia in mice. Neuroreport. 1999;10(1):47-51.

57. Schwarzkopf TM, Hagl S, Eckert GP, et al. Neuroprotection by bilobalide in ischemia: improvement of mitochondrial function. Pharmazie. 2013;68(7):584-589.

58. Ahlemeyer B, Krieglstein J. Neuroprotective effects of Ginkgo biloba extract. Cell Mol Life Sci. 2003;60(9):1779-1792.

59. Li ZY, Chung YH, Shin EJ, et al. YY-1224, a terpene trilactonestrengthened Ginkgo biloba, attenuates neurodegenerative changes induced by $\beta$-amyloid (1-42) or double transgenic overexpression of APP and PS1 via inhibition of cyclooxygenase-2. J Neuroinflam. 2017;14(1):94-116.

60. Wu Y, Sun J, George J, et al. Study of neuroprotective function of Ginkgo biloba extract (EGb761) derived-flavonoid monomers using a three-dimensional stem cell-derived neural model. Biotechnol Prog. 2016;32(3):735-744. 
61. Coward L, Barnes NC, Setchell KDR, et al. Genistein, daidzein, and their $\beta$-glycoside conjugates: antitumor isoflavones in soybean foods from American and Asian diets. J Agr Food Chem. 1993;41(11):1961-1967.

62. Kaufman PB, Duke JA, Brielmann H, et al. A comparative survey of leguminous plants as sources of the isoflavones, genistein and daidzein: implications for human nutrition and health. J Altern Complement Med. 1997;3(1):7-12.

63. Alves RC, Almeida IM, Casal S, et al. Isoflavones in coffee: influence of species, roast degree, and brewing method. J Agric Food Chem. 2010;58(5):3002-3007.

64. Rao HSP, Reddy KS. Isofavones from Flemingia vestita. Fitoterapia 1991;62:458-460.

65. Cui S, Wang J, Wu Q, et al. Genistein inhibits the growth and regulates the migration and invasion abilities of melanoma cells via the FAK paxillin and MAPK pathways. Oncotarget. 2017;8(13):21674-21691.

66. Sung MJ, Kim DH, Davaatseren M, et al. Genistein suppression of TNFalpha-induced fractalkine expression in endothelial cells. Cell Physiol Biochem. 2010;26(3):431-440.

67. Hwang JT, Lee YK, Shin JI, et al. Anti-inflammatory and anticarcinogenic effect of genistein alone or in combination with capsaicin in TPA-treated rat mammary glands or mammary cancer cell line. Ann N Y Acad Sci. 2009; 1171:415-420.

68. Harper CE, Cook LM, Patel BB, et al. Genistein and resveratrol, alone and in combination, suppress prostate cancer in SV-40 tag rats. Prostate. 2009;69(15):1668-1682.

69. De Paula ML, Rodrigues DH, Teixeira HC, et al. Genistein downmodulates pro-inflammatory cytokines and reverses clinical signs of experimental autoimmune encephalomyelitis. Int Immunopharmacol. 2008;8(9):1291-1297.

70. Nilsson S, Mäkelä S, Treuter E, et al. Mechanisms of estrogen action. Physiol Rev. 2007;81(4):1535-1565.

71. Harris HA. Estrogen receptor-beta: recent lessons from in vivo studies. Mol Endocrinol. 2007;21(1):1-13.

72. Arce C, Arteaga JL, Eduardo SM, et al. Added after Anoxia-Reoxigenation Stress, Genistein Rescues from Death the Rat Embryo Cortical Neurons. Neuros Med. 2010;1(2):50-59.

73. Valsecchi AE, Franchi S, Panerai AE, et al. Genistein, a natura phytoestrogen from soy relieves neuropathic pain following cheonic constriction sciatic nerve injury in mice: anti-inflammatory and antioxidant activity. $J$ Neurochem. 2001;107(1):230-240.

74. Song TT, Hendrich S, Murphy PA. Estrogenic activity of glycitein, a soy isoflavone. J Agr Food Chem. 1999;47(4):1607-1610.

75. Sacks FM, Lichtenstein A, Van Horn L, et al. Soy Protein, Isoflavones, and Cardiovascular Health: An American Heart Association Science Advisory for Professionals from the Nutrition Committee. Circulation 2006;113(7):1034-1044.

76. Burguete MC, Torregrosa G, Pérez-Asensio FJ, et al. Dietary Phytoestrogens Improve Stroke Outcome after Transient Focal Cerebral Ischemia in Rats. Eur J Neurosci. 2006;23(3):703-710.

77. File SE, Hartley DE, Alom N, et al. Soya Phytoestrogens Change Cortical and Hippocampal Expression of BDNF mRNA in Male Rats. Neuros Lett. 2003;338(2):135-138.

78. Woo J, Lynn H, Lau WY, et al. Nutrient intake and psychological health in an elderly Chinese population. Int $J$ Geriatr Psychiatry. 2006;21(11):1036-1043.

79. Kreijkamp-Kaspers S, Kok L, Grobbee DE, et al. Dietary phytoestrogen intake and cognitive function in older women. J Gerontol A Biol Sci Med Sci. 2007;62(5):556-562.
80. White LR, Petrovitch H, Ross GW, et al. Brain aging and midlife tofu consumption. J Am Coll Nutr. 2000;19(2):242-255.

81. Malinowska M, Wilkinson FL, Langford-Smith KJ, et al. Genistein Improves Neuropathology and Corrects Behaviour in a Mouse Model of Neurodegenerative Metabolic Disease. PLOS One. 2010;5(12):1-8.

82. Akiyama T, Ishida J, Nakagawa S, et al. Genistein, a specific inhibitor of tyrosine-specific protein kinases. J Biol Chem. 1987;262(12):5592-5595.

83. McClain RM, Wolz E, Davidovich A, et al. Reproductive safety studies with genistein in rats. Food Chem Toxicol. 2007;45(8):1319-1332.

84. Piotrowska E, Jakóbkiewicz-Banecka J, Barańska S, et al. Genisteinmediated inhibition of glycosaminoglycan synthesis as a basis for gene expression-targeted isoflavone therapy for mucopolysaccharidoses. Eur $J$ Hum Genet. 2006;14(7):846-852.

85. Malinowska M, Wilkinson FL, Bennett W, et al. Genistein reduces lysosomal storage in peripheral tissues of mucopolysaccharide IIIB mice. Mol Genet Metab. 2009;98(3):235-242.

86. Kong LY, Lai C, Wilson BC, et al. Protein tyrosine kinase inhibitors decrease lipopolysaccharide-induced proinflammatory cytokine production in mixed glia, microglia-enriched or astrocyte-enriched cultures. Neurochem Int. 1997;30(4-5):491-497.

87. Wang X, Chen S, Ma G, et al. Genistein protects dopaminergic neurons by inhibiting microglial activation. Neuroreport. 2005;16(3):267-270.

88. Moskot M, Montefusco S, Jakóbkiewicz-Banecka J, et al. The Phytoestrogen Genistein Modulates Lysosomal Metabolism and Transcription Factor EB (TFEB) Activation. $J$ Biol Chem. 2014;289(24):17054-17069.

89. Sardiello M, Palmieri M, di Ronza A, et al. A gene network regulating lysosomal biogenesis and function. Science. 2009;325(5939):473-477.

90. Ren B, Liu Y, Zhang Y, et al. Genistein: A Dual Inhibitor of Both Amyloid $\beta$ and Human Islet Amylin Peptides. ACS Chem Neurosci. 2018;9(5):1215-1224.

91. Park YJ, Ko JW, Jeon S, et al. Protective Effect of Genistein against Neuronal Degeneration in ApoE-/- Mice Fed a High-Fat Diet. Nutrients. 2016;8(11):692-703

92. Profenno LA, Porsteinsson AP, Faraone SV. Meta-analysis of Alzheimer's disease risk with obesity, diabetes, and related disorders. Biol Psychiatry. 2010;67(6):505-512.

93. Poirier J. Apolipoprotein E and Alzheimer's disease. A role in amyloid catabolism. Ann N Y Acad Sci. 2000;924:81-90.

94. Yu HL, Li L, Zhang XH, et al. Neuroprotective effects of genistein and folic acid on apoptosis of rat cultured cortical neurons induced by $\beta$-amyloid 31-35. Brit J Nut. 2009;102(5):655-662.

95. Xi YD, Zhang DD, Ding J, et al. Genistein Inhibits A $325-35$-Induced Synaptic Toxicity and Regulates CaMKII/CREB Pathway in SH-SY5Y Cells. Cell Mol Neurobiol. 2016;36:1151-1159.

96. McDowell ML, Das A, Smith JA, et al. Neuroprotective effects of genistein in VSC4.1 motoneurons exposed to activated microglial cytokines. Neurochem Int. 2011;59(2):175-184.

97. Ma W, Ding B, Yu H, et al. Genistein Alleviates $\beta$-Amyloid-Induced Inflammatory Damage Through Regulating Toll-Like Receptor 4/Nuclear Factor kB. J Med Food. 2015;18(3):273-279.

98. Ma WW, Hou CC, Zhou X, et al. Genistein alleviates the mitochondriatargeted DNA damage induced by $\beta$-amyloid peptides 25-35 in C6 glioma cells. Neurochem Res. 2013;38(7):1315-1323.

99. Liu LX, Chen WF, Xie JX, et al. Neuroprotective effects of genistein on dopaminergic neurons in the mice model of Parkinson's disease. Neurosci Res. 2008;60(2):156-161. 